\title{
EDITORIAL
}

\section{Comparison of acute myeloid leukemia patients at MD Anderson: 1982-1986 vs 1992-1996}

\author{
PM Hoff, S Pierce and E Estey \\ Department of Hematology, Section of Leukemia, The University of Texas MD Anderson Cancer Center, Houston, TX, USA
}

\begin{abstract}
We compared the acute myeloid leukemia (AML) population seen at UT MD Anderson Cancer Center in the period from 1982 to 1986 with the population seen in the period of 1992 to 1996. The population seen in the 1990 s was significantly $(P<0.01)$ older than the population seen in the 1980 s, and was more likely $(P<0.001)$ to have abnormalities of chromosomes 5 and/or (5q-, -5, 7q-, -7). The increase in the incidence of $-5 /-7$ was seen in both younger and older patients. Because the populations differed in these ways it is likely that they differed in other, less quantifiable ways. Our results suggest caution when using historical controls and when generalizing findings from randomized studies.
\end{abstract}

Keywords: acute myelogenous leukemia; age; historical control; $-5 ;-7$; karyotype

\section{Background}

In an attempt to better compare outcomes with different therapies, physicians have identified several prognostic factors in patients with newly diagnosed acute myeloid leukemia (AML). Among these are karyotype, age, performance status, organ function, prior exposure to chemotherapy or radiotherapy and antecedent hematological disorder. ${ }^{1-4}$ The last decade has witnessed important changes in the structure of health care in the United States, ${ }^{5,6}$ an aging of the population, and perhaps a greater exposure to carcinogens. ${ }^{7,8}$ To assess the influence of these changes we used prognostic factors such as those described above to compare patients with newly diagnosed AML who presented to MD Anderson for treatment from 1982 to $1986\left({ }^{\prime} 82-86^{\prime}\right)$ with those who presented from 1992 to 1996 ('92-96').

\section{Materials and methods}

We saw 324 patients who met FAB criteria for AML in the period 82-86, and 501 in the period 92-96. We compared the age, leukemia cell karyotypes, Zubrod performance status, FAB type, and presence of antecedent hematological disorder. Antecedent hematological disorder was defined as a documented abnormality in blood count for more than 1 month prior to presentation to MD Anderson. Categorical data were compared using the $\chi^{2}$ and continuous data compared using the Kruskal-Wallis test. Given the multiple comparisons, we considered $P$ values $<0.01$ as statistically significant. Patients are almost always referred by their primary physicians to our institution for treatment, rather than a 'second opinion'. These

Correspondence: E Estey, Department of Hematology, Box 61, UT MD Anderson Cancer Center, 1515 Holcombe Blvd, Houston, Texas 77030, USA; Fax: 7137944297

Received 7 July 1997; accepted 22 August 1997 patients received a variety of different therapeutic combinations, based on their prognosis at presentation.

\section{Results}

We observed an increase in the age of the more recent population $(P<0.01)$. The last column of Table 1 illustrates that the 25th, 50th and 75th percentiles of the age distribution each increased by 5-7 years between 82-86 and 92-96. In general, the patients presenting in any given year in the 9296 period were older than those presenting in any given year in the 82-86 period. Further analysis indicates that the aging of our population resulted from an increase in the number of older patients rather than a decrease in the number of younger ones. There were 253 patients less than 64 years of age in the 82-86 group against 334 in the 92-96 group (a 1.3-fold increase), while there were 81 older than 64 years of age in the 82-86 group against 167 in the 92-96 group (a 2.1-fold increase). There was also an increase in patients presenting with abnormalities of chromosomes 5 and/or 7 and a corresponding decrease in those presenting with a normal karyotype (Table 2). Abnormalities in chromosomes 5 and/or 7, of course, are well-known to be associated with poor prognosis. When analyzed separately, both age groups (above and below 64 years of age) showed an increase in the incidence of these unfavorable karyotypes with statistically significant results ( $P=0.019$ for the younger, and $P=0.047$ for the older patients). There was no significant difference in the proportion of patients with favorable performance status (Zubrod of 0 2 as opposed to 3-4), or in the incidence of an antecedent hematological disorder.

Although a trivial prognostic factor once cytogenetics, antecedent hematological disorder, age and performance status have been accounted for, the FAB subtypes of $A M L$ underwent significant changes. There were fewer cases of $M 2(26.2 \%$ in $82-86$ and $16.6 \%$ in $92-96)$ and M5 (10.2-5.4\%) and more of $\mathrm{M} 6(0.9-3.6 \%)$ and $\mathrm{M} 7(0-3.2 \%)$. Complete response rates were essentially indistinguishable during the two time periods (63\% for $82-86,65 \%$ for $92-96)$, as were disease-free survival (median of 21 weeks for both 82-86 and 92-96) and survival (median of 42 weeks for 82-86 and 50 weeks for 92-96).

\section{Discussion}

A principal finding of our paper is the increasingly elderly nature of patients seen in the 92-96 period compared to patients seen a decade before. Our patients are mainly referred by outside physicians and consequently the demographics of our population could be influenced by changes in the referral pattern. Given that the absolute number of younger patients has not changed significantly, it does not appear that physicians 
25th, 50th and 75th percentile of age distribution between 1982-1986 and 1992-1996

\begin{tabular}{|c|c|c|c|c|c|c|}
\hline & $82 / 92^{a}$ & $83 / 93$ & $84 / 94$ & $85 / 95$ & $86 / 96$ & $82-86 / 92-96^{b}$ \\
\hline 25th percent & $32 / 33$ & $35 / 40$ & $31 / 38$ & $32 / 42$ & $36 / 43$ & $33 / 40$ \\
\hline 50th percent & $52 / 51$ & $50 / 58$ & $52 / 54$ & $46 / 58$ & $52 / 60$ & $50 / 57$ \\
\hline 75th percent & $62 / 69$ & $63 / 70$ & $65 / 64$ & $61 / 70$ & $64 / 69$ & $63 / 68$ \\
\hline No. patients & $73 / 99$ & $55 / 93$ & $53 / 103$ & $68 / 105$ & $75 / 101$ & $324 / 501$ \\
\hline
\end{tabular}

aComparison of 1982 with 1992.

${ }^{\mathrm{b}}$ Comparison of 1982-1986 with 1992-1996.

Table 2 Karyotype distribution: 1982-1986 vs 1992-1996

\begin{tabular}{lccr}
\hline & $82-86(\%)$ & $92-96(\%)$ & $P$ value \\
\hline Normal & $131(40.4)$ & $137(27.3)$ & $<0.001$ \\
t(8;21) & $17(5.2)$ & $17(3.4)$ & 0.187 \\
t(15;17) & $21(5.2)$ & $44(8.8)$ & 0.234 \\
Inversion 16 & $16(4.9)$ & $20(4)$ & 0.516 \\
+8 & $25(7.7)$ & $36(7.2)$ & 0.772 \\
$-5 /-7$ & $37(11.4)$ & $102(20.4)$ & $<0.001$ \\
$11 q-$ & $11(3.4)$ & $26(5.2)$ & 0.230 \\
Miscellaneous & $34(10.5)$ & $77(15.4)$ & 0.046 \\
Insufficient for analysis & $29(9)$ & $38(7.6)$ & 0.472 \\
& & & \\
Total & $321^{\mathrm{a}}$ & $497^{\mathrm{b}}$ & \\
\hline
\end{tabular}

${ }^{a}$ Not done in three patients.

bNot done in four patients.

are simply referring fewer younger patients. Rather, and consistent with the general aging of the population, it appears more likely that the rates of referral for younger and elder patients could be unchanged, but that there could be more older patients with AML in the population from which our referrals are drawn. The absolute increase in patients with abnormalities of chromosomes 5 and/or 7 occurred in all age groups, and does not reflect purely the increasing number of elderly patients as one could assume. Our experience indicates that physicians usually do not obtain cytogenetics before referring a patient, and thus cytogenetic findings would have little impact in their decision to refer the patient for treatment.

A precondition for conducting historically controlled studies is the assurance that the current and historical populations are compatible. If they differed only with respect to age and cytogenetics, the differences between historical and current population could be dealt with using multivariate regression. Because, however, the populations differed in both these characteristics, it is more likely that they also differed with regard to other, unknown, independent characteristics than would be the case if the 82-86 and 92-96 populations were identical. Although unknown, these characteristics could be prognostic. Simon ${ }^{9}$ has pointed out that known prognostic factors account for only $20-25 \%$ of the variability in patient out- come. The unaccounted variability could be explained by random phenomena or equally likely by the existence of unknown prognostic factors. Thus, the differences between the 82-86 and 92-96 population raise questions as to the validity of comparison of treatment in these populations and, by extension, in other populations involving historical controls. These considerations may also apply to interpretation of randomized studies. Indeed, Bailey ${ }^{10}$ has pointed out the difficulties in generalizing the results of such studies to populations at different risks.

\section{References}

1 Estey EH, Keating MJ, McCredie KB, Bodey G, Freireich E. Causes of initial remission failure in acute myelogenous leukemia. Blood 1982; 60: 309-315.

2 Keating MJ, Smith T, Kantarjian H, Cork A, Walters R, Trujillo JM, McCredie KB, Gehan EA, Freireich EJ. Cytogenetic pattern in acute myelogenous leukemia: a major reproducible determinant of outcome. Leukemia 1988; 2: 403-412.

3 Rai KR, Holland JF, Glidewell OJ, Weinberg V, Brunner K, Obrecht JP, Preisler HD, Nawabi IW, Prager D, Carey RW, Cooper MR, Haurani F, Hutchinson JL, Silver RT, Falkson G, Wiernik P, Hoagland HC, Bloomfield CD, James GW, Gottlieb A, Ramanan SV, Blom J, Nissen NI, Bank A, Ellison RR, Kung F, Henry P, Mclntyre OR, Kaan SK. Treatment of acute myelocytic leukemia: a study by Cancer and Leukemia Group B. Blood 1981; 58: 1203-1212.

4 Preisler HD, Raza A, Barcos M, Azarnia N, Larson R, Walker I, Browman M, Grunwald H, D'Arrigo P, Doeblin T, Bloom M, Stein A, Logue G, Goldberg J, Kirshner J, Gottlieb A, Bennett J. High dose cytosine arabinoside as the initial treatment of poor risk patients with acute nonlymphocytic leukemia: a leukemia intergroup study. J Clin Oncol 1987; 5: 75-82.

5 Lichiello P, Madden CW. Context and catalysts for change in health care markets. Health Aff 1996; 15; 121-129.

6 Blumenthal D, Meyer GS. Academic health centers in a changing environment. Health Aff 1996; 15: 200-215.

7 Parker SL, Tong T, Bolden S, Wingo PA. Cancer statistics, 1997. Cancer J Clin 1997; 47: 5-27.

8 Cunningham MP. Giving life to numbers. CA J Clin 1997; 47: 3-4.

9 Simon R. Importance of prognostic factors in cancer clinical trials. Cancer Treat Rep 1984; 68: 185-192.

10 Bailey KR. Generalizing the results of randomized clinical trials. Controlled Trials 1994; 15: 15-23. 